\title{
Antiferroelectric-Like Behavior of Vinylidene Fluoride/Trifluoroethylene Copolymers with Low Vinylidene Fluoride Content
}

\author{
Yoshiyuki Takahashi, Hidekazu Kodama, Masayuki NaKamura, \\ Takeo Furukawa, and Munehiro Date* \\ Department of Chemistry, Faculty of Science, Science University of Tokyo, \\ 1-3 Kagurazaka, Shinjuku, Tokyo 162-8601, Japan \\ * The Institute of Physical and Chemical Research \\ 2-1 Hirosawa, Wako, Saitama 351-0198, Japan
}

(Received August 10, 1998)

\begin{abstract}
Vinylidene Fluoride/Trifluoroethylene copolymers with compositions $47 / 53$ and $37 / 63 \mathrm{~mol} \%$ exhibit a ferroelectric square $D-E$ hysteresis loop at low temperatures (below $-40^{\circ} \mathrm{C}$ ) and undergo transition to a paraelectric motional phase near $T_{\mathrm{c}}=70^{\circ} \mathrm{C}$. At intermediate temperature they exhibit a double-hysteresis loop suggesting that polarization reversal occurs through a non-polar phase. Time domain measurement of polarization reversal shows two-step switching transient consisting of fast depolarization and slow re-polarization processes. The remanent polarization imparted in the ferroelectric phase shows gradual decay on heating and disappears completely well below $T_{\mathrm{c}}$. We conclude that these copolymers take an antiferroelectric-like non-polar phase between low temperature ferroelectric and high temperature paraelectric phases.

KEY WORDS Vinylidene Fluoride/Trifluoroethylene Copolymer / Ferroelectricity / Antiferroelectricity Double Hysteresis / Switching / Depolarization /
\end{abstract}

Copolymers of vinylidene fluoride (VDF) with trifluoroethylene $(\mathrm{TrFE})$ have attracted much attention for the past fifteen years because of their ferroelectric properties and associated piezoelectric and pyroelectric activities. ${ }^{1-3}$ Of special importance are copolymers containing $50-80 \mathrm{~mol} \%$ VDF which exhibit fast polarization reversal as well as a clear Curie point $T_{\mathrm{c}}$. When VDF content exceeds $80 \mathrm{~mol} \%, T_{\mathrm{c}}$ is not observable at atmospheric pressure because it is overtaken by melting. When TrFE becomes a major component, ferroelectricity is obscured and finally disappears in polytrifluoroethylene.

The ferroelectric phase of a VDF-rich copolymer consists of all-trans molecules packed in parallel to each other in a pseudo-hexagonal lattice. In the paraelectric phase, molecules undergo active rotational motion by adopting a random sequence of TT, TG, and $\mathrm{TG}^{\prime}$ bonds. Thus, spontaneous polarization is lost intramolecularly. The transition is of first order judging from thermal hysteresis. The $70 / 30 \mathrm{~mol} \% \mathrm{VDF} / \mathrm{TrFE}$ copolymer has $T_{\mathrm{c}}$ at $110^{\circ} \mathrm{C}$ on heating and $70^{\circ} \mathrm{C}$ on cooling. As VDF content decreases to $50 \mathrm{~mol} \%$, thermal hysteresis is lost and the transition becomes second-order.

A copolymer with equi-molar composition is complex in that it takes a diffuse phase when cooled from the paraelectric phase. The X-ray diffraction profile associated with $(110) /(200)$ interchain reflections consists of broad double peaks. Tashiro et al. ${ }^{3}$ designated such a phase as the "cooled phase" and assigned it to an aggregate of tilted monoclinic crystalline domains connected by gauche or skew bonds. Lovinger et al. ${ }^{4}$ and Horiuchi et al. ${ }^{5}$ proposed a two-phase structure consisting of well-ordered and less-ordered pseudohexagonal phases. In any case, such a diffuse phase is transformed into a regular ferroelectric phase showing a single $(110) /(200)$ reflection peak by applying a suf- ficiently high electric field if VDF content is slightly larger than $50 \mathrm{~mol} \%$. When VDF content becomes smaller than $50 \mathrm{~mol} \%$, the diffuse phase is maintained and ferroelectricity is obscured.

Koizumi et al. ${ }^{6}$ reported VDF/TrFE copolymers with VDF content smaller than $50 \mathrm{~mol} \%$ to exhibit a $D-E$ double hysteresis loop, implying a non-polar state between positively and negatively polarized states. They found $^{7}$ that X-ray diffraction $(110) /(200)$ changed from a double to single peak when a high electric field was applied and that the structure which gave a double peak corresponds to a non-polar and a single peak to the polar state.

This paper examines $\mathrm{VDF} / \mathrm{TrFE}$ copolymers with composition $47 / 53$ and $37 / 63 \mathrm{~mol} \%$. We undertook switching measurements to obtain information on polarization reversal including dynamical characteristics. We made thermal depolarization and dielectric measurements for samples poled in the ferroelectric phase. The results are discussed in relation to antiferroelectricity.

\section{EXPERIMENTAL}

The samples used were random copolymers of VDF and TrFE with composition $47 / 53$ and $37 / 63 \mathrm{~mol} \%$ from Daikin Industries, Ltd. Films cast from dimethylformamide were uniaxially-drawn 5-7 times the original length at room temperature. After annealing at $150^{\circ} \mathrm{C}$ for an hour, gold was evaporated on both surfaces to serve as electrodes.

$D-E$ hysteresis loops were obtained by measuring charge response induced by application of a sinusoidal voltage at $0.1 \mathrm{~Hz}$. Switching curves were obtained by applying step-wise voltage and measuring charge response as a function of time. After repetitive applica-

\footnotetext{
† Present address: Nippon Zeon Co., Ltd., 1-2-1 Yako, Kawasaki, Kawasaki 210-0863, Japan.
} 


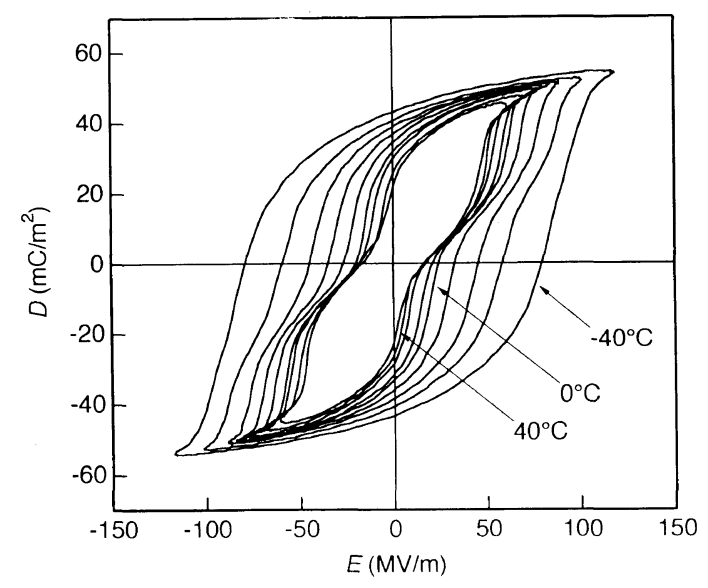

Figure 1. $D-E$ hysteresis loops of a $\operatorname{VDF}(47) / \operatorname{TrFE}(53)$ copolymer at $-40^{\circ} \mathrm{C}$ to $40^{\circ} \mathrm{C}$.

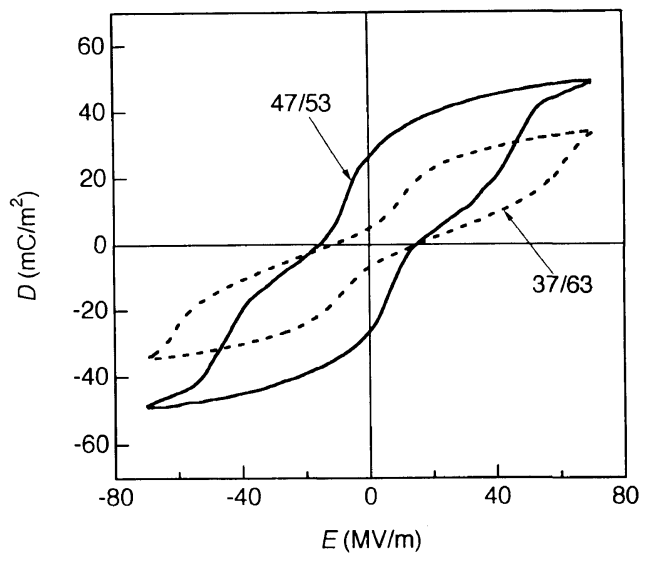

Figure 2. Comparison of shapes of double hysteresis loops of $\operatorname{VDF}(47) / \operatorname{TrFE}(53)$ and $\operatorname{VDF}(37) / \operatorname{TrFE}(63)$ copolymers at $20^{\circ} \mathrm{C}$.

tions of step voltages with alternating polarity, reverse switching curve was obtained by applying a voltage with polarity opposite to the last one. The forward switching curve was obtained using voltage with the same polarity. Temperature was varied from $-80^{\circ} \mathrm{C}$ to $80^{\circ} \mathrm{C}$ in $D-E$ hysteresis and switching measurements.

Depolarization of a poled sample was measured by a charge amplifier at a heating rate of $2 \mathrm{~K} \mathrm{~min}^{-1}$. Dielectric measurement was made for the same sample at $1 \mathrm{kHz}$ using an impedance analyzer (HP 4284A).

\section{RESULTS AND DISCUSSION}

\section{D-E Hysteresis Loop}

Figure 1 shows $D-E$ hysteresis loops for the $\operatorname{VDF}(47)$ $\operatorname{TrFE}(53)$ copolymer at various temperatures. At $-40^{\circ} \mathrm{C}$, a single square loop substantiating ferroelectricity was found. As temperature is increased to room temperature, the loop is transformed into a propeller-type double loop. This indicates that polarization reversal occurs in two steps: the first associated with depolarization of saturated polarization and the second, associated with repolarization in the opposite direction.

The VDF(37)/TrFE(63) copolymer exhibits essentially the same behavior except that double loop character is more pronounced. Figure 2 shows a comparison of hysteresis loops at room temperature. When the applied



Figure 3. Switching characteristics of a $\operatorname{VDF}(47) / \operatorname{TrFE}(53)$ copolymer at $25^{\circ} \mathrm{C}$ at $30 \mathrm{MV} \mathrm{m}^{-1}$ to $80 \mathrm{MV} \mathrm{m}^{-1}$.

electric field crosses zero, the $37 / 63 \mathrm{~mol} \%$ copolymer returns nearly to a non-polar state whereas the $47 / 53$ mol\% copolymer remains in a polar state, as noted by Koizumi et al. ${ }^{6}$

\section{Switching Measurement}

To confirm two-step polarization reversal and gain further information about dynamical characteristics, we undertook switching measurements. The results are shown in Figure 3 where electric displacement $D$ is plotted against the logarithm of time $t$ after application of a step field from $30 \mathrm{MV} \mathrm{m}^{-1}$ to $80 \mathrm{MV} \mathrm{m}^{-1}$ for the $47 / 53 \mathrm{~mol} \%$ copolymer at $25^{\circ} \mathrm{C}$. Prior to measurement, the sample was subjected to a field of $80 \mathrm{MV} \mathrm{m}^{-1}$ in the opposite direction. The origin of ordinate $(D=0)$ thus corresponds to the state where the sample has a negative remanent polarization. At $40-60 \mathrm{MV} \mathrm{m}^{-1}$, we find a two-step increment of $D$ implying that depolarization occurs first and then re-polarization. As the strength of the applied field increases, the curve moves to a short time region. The second step depends on the applied field more strongly than the first and thus become inseparable above $80 \mathrm{MV} \mathrm{m}^{-1}$.

Figure 4 shows switching characteristics of the same copolymer at $0,-20$ and $-40^{\circ} \mathrm{C}$ under a field of 80 $\mathrm{MV} \mathrm{m}^{-1}$. The two-step process is transferred to a single process as temperature decreases to $-40^{\circ} \mathrm{C}$. This is consistent with the transition of the $D-E$ relation from a double to single loop at the same temperature. In the figure, the derivative of $D$ with respect to $\log t$ is plotted. From the peak position, we determine time constants $\tau_{1}$ and $\tau_{2}$ of depolarization and re-polarization, respectively.

Figure 5 shows plots of $\log \tau_{1}$ and $\log \tau_{2}$ against the reciprocal of applied field $1 / E$. The linear relationship indicates these time constants obey

$$
\tau=\tau_{0} \exp \left(E_{\mathrm{a}} / E\right)
$$

which has been shown to hold for the ferroelectric switching time in VDF-rich copolymers. ${ }^{8} \tau_{0}$ is the time constant at infinite electric field and $E_{\mathrm{a}}$ the activation field. From the slope of the linear relationship, we obtain $E_{\mathrm{a}}$ of $0.28 \mathrm{GV} \mathrm{m}^{-1}$ for depolarization and $0.83 \mathrm{GV} \mathrm{m}^{-1}$ for re-polarization at $25^{\circ} \mathrm{C}$. Because of such difference in $E_{\mathrm{a}}$, these two steps merge at high field of $c a .100$ $\mathrm{MV} \mathrm{m}^{-1}$. As temperature decreases, $\tau_{1}$ increases more rapidly than $\tau_{2}$. Thus, these time constants coincide to a 


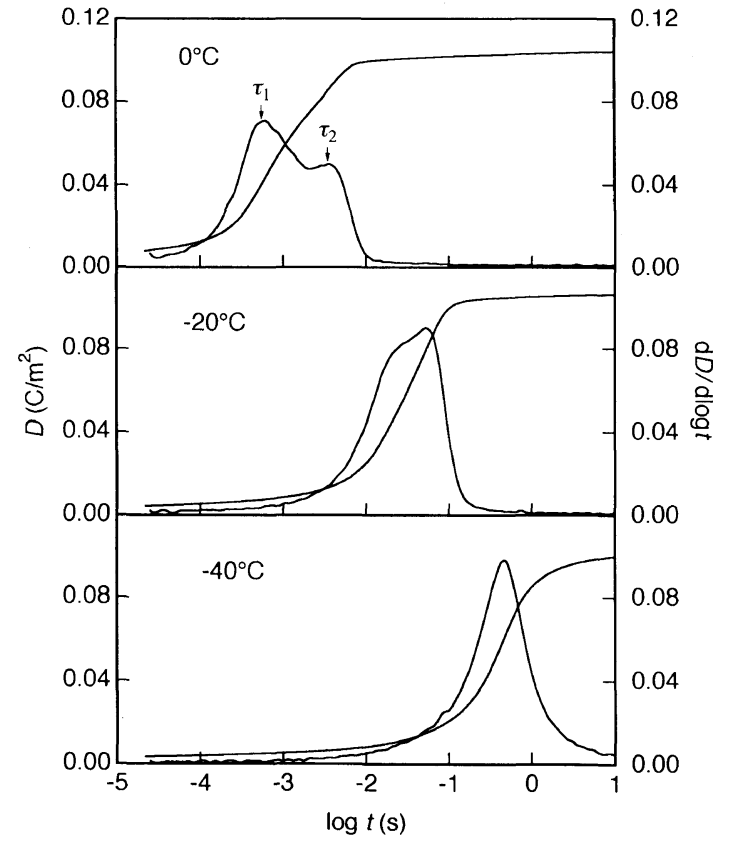

Figure 4. Switching characteristics of a $\operatorname{VDF}(47) / \operatorname{TrFE}(53)$ copolymer at $0,-20$ and $-40^{\circ} \mathrm{C}$ at $80 \mathrm{MVm}^{-1}$

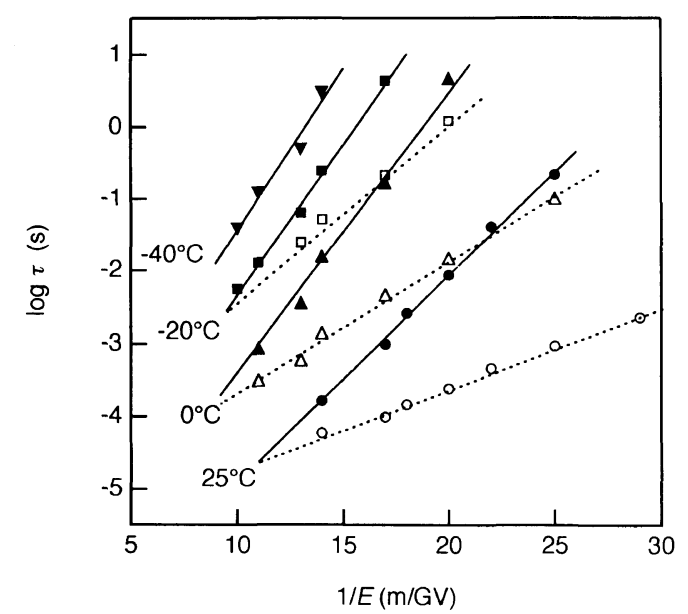

Figure 5. Plots of logarithms of time constants associated with depolarization $\left(\tau_{1}\right.$, open marks) and re-polarization $\left(\tau_{2}\right.$, filled marks $)$ in a $\operatorname{VDF}(47) / \operatorname{TrFE}(53)$ copolymer.

single process at $-40^{\circ} \mathrm{C}$.

The $37 / 63 \mathrm{~mol} \%$ copolymer exhibited a two-step process. As shown by the double hysteresis loop in Figure 2 , this copolymer undergoes partial depolarization when the applied field crosses zero. This is quantitatively demonstrated in Figure 6 in terms of difference in reverse and forward switching curves at $10^{\circ} \mathrm{C}$ at $60 \mathrm{MV} \mathrm{m}^{-1}$. The reverse switching curve $D_{\mathrm{R}}$ (solid line) showed a two-step character associated with depolarization of remaining polarization and re-polarization in the direction of the applied field. The time constants $\tau_{1}$ and $\tau_{2}$ of respective steps were evaluated from positions of $\mathrm{d} D_{\mathrm{R}} / \mathrm{d} \log t$ double peaks. The forward switching curve $D_{\mathrm{F}}$ (dashed line) revealed only re-polarization of the depolarized component with time constant $\tau_{\mathrm{F}}$. This component was not clearly observed in the $47 / 53$ copolymer because it mostly stayed in a polarized state when the applied field was removed.

Increments of polarization $\Delta D_{\mathrm{R}}$ and $\Delta D_{\mathrm{F}}$ evaluated

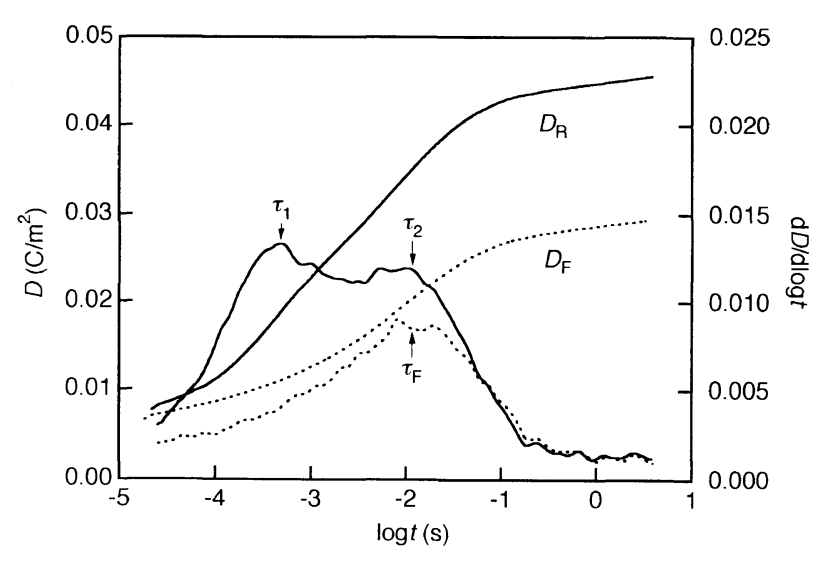

Figure 6. Switching characteristics of a $\operatorname{VDF}(37) / \operatorname{TrFE}(63)$ copolymer obtained by reverse $\left(D_{\mathrm{R}}\right.$, solid curves) and forward $\left(D_{\mathrm{F}}\right.$, dashed curves) measurements.

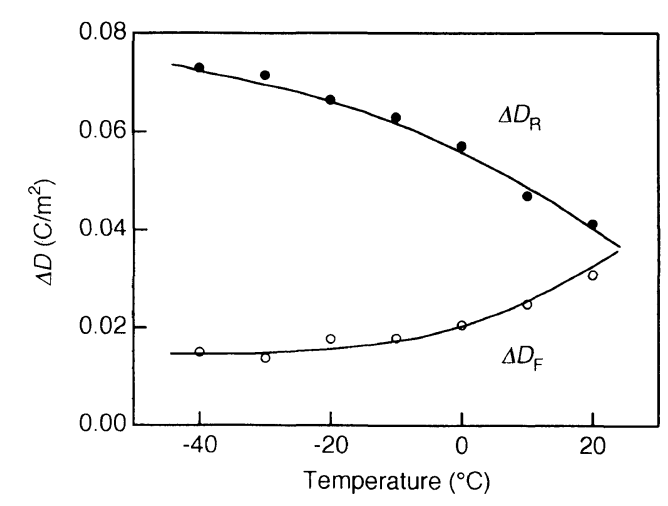

Figure 7. Increments of polarization during reverse $\left(\Delta D_{\mathrm{R}}\right.$ : solid line $)$ and forward $\left(\Delta D_{\mathrm{F}}\right.$ : dashed line) switching measurements at various temperatures for a $\operatorname{VDF}(37) / \operatorname{TrFE}(63)$ copolymer.

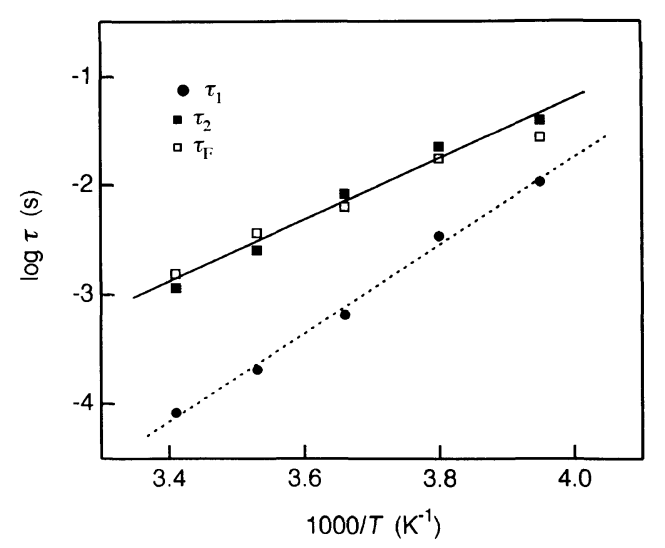

Figure 8. Temperature dependence of switching times $\tau_{1}, \tau_{2}$, and $\tau_{\mathrm{F}}$ for a $\operatorname{VDF}(37) / \operatorname{TrFE}(63)$ copolymer at $80 \mathrm{MV} \mathrm{m}^{-1}$.

from the value of $D$ at $t=3 \mathrm{~s}$ in Figure 6 are plotted in Figure 7 as a function of temperature. As temperature increases, $\Delta D_{\mathrm{R}}$ decreases whereas $\Delta D_{\mathrm{F}}$ increases because depolarization under a short-circuit condition is more pronounced. At $20^{\circ} \mathrm{C}, \Delta D_{\mathrm{R}}$ coincides with $\Delta D_{\mathrm{F}}$ implying full depolarization.

Time constants $\tau_{1}, \tau_{2}$, and $\tau_{\mathrm{F}}$ of the $37 / 63 \mathrm{~mol} \%$ copolymer depend on the applied field according to an exponential law analogous to the $47 / 53 \mathrm{~mol} \%$ copolymer and on temperature as shown in Figure 8 where the logarithms of the time constants are plotted against the 


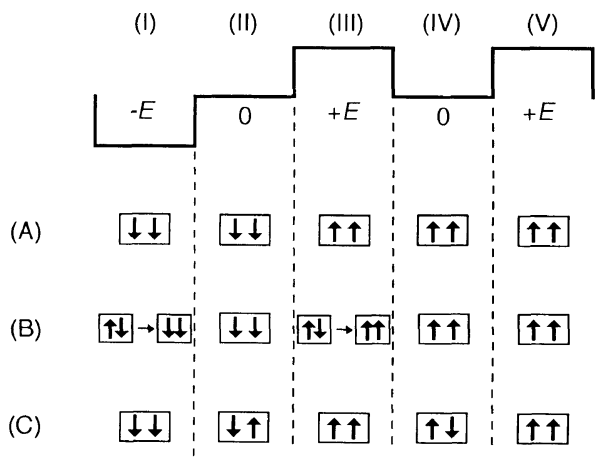

Figure 9. Schematic representation of changes in polarization states during switching measurements in (A) ferroelectric and (C) antiferroelectric substances. (B) illustrates two step switching.

reciprocal of absolute temperature $1 / T$. The strength of the applied field was fixed at $80 \mathrm{MV} \mathrm{m}^{-1}$. Linear relationship suggests these processes are activated not only electrically but also thermally. From the slope, we obtain an activation energy of $78 \mathrm{~kJ} \mathrm{~mol}^{-1}$ for depolarization and $48 \mathrm{~kJ} \mathrm{~mol}^{-1}$ for re-polarization. $\tau_{\mathrm{F}}$ coincides with $\tau_{2}$, because both are concerned with re-polarization.

VDF/TrFE copolymers with composition 47/53 and $37 / 63 \mathrm{~mol} \%$ exhibit two-step switching due to non-polar state. Figure 9 summarizes changes in polarization during switching. On the top is shown the profile of the applied electric field in five stages, $-E$ (I), 0 (II), $+E$ (III), 0 (IV) and $+E$ (V). Prior to measurement, the sample was subjected to a negative field (I) and then to a zero-field (II) for certain period, typically 100 s. Reverse and forward measurements were made at stages (III) and (V), respectively. Arrows in the same direction express saturated polarization and in the opposite direction, no polarization.

Case (A) represents a typical ferroelectric in that polarization is reversed when the electric field is applied in the direction opposite to the remanent polarization (III). Reversed polarization remains unchanged when the field is removed (IV). Therefore, subsequent forward measurement $(\mathrm{V})$ yields only minor polarization change associated with reversible dielectric response. For (C), saturation polarization is induced by an electric field but is lost completely at a zero-field. This is known to be the case of typical antiferroelectric. Case (B) represents two-step switching in that polarization reversal occurs through a non-polar state.

In $47 / 53$ and $37 / 63$ copolymers a combination of these three cases were observed depending upon the strength of applied field and the temperature. At low temperatures, they exhibited typical ferroelectric switching (A). As the temperature increased, the two-step character (B) became dominating but partial depolarization at zero-field (C) occurred. Above room temperature, the $37 / 63$ copolymer exhibited full depolarization and single re-polarization. Cases (B) and (C) clearly indicate these copolymers to be in a non-polar state as a stable or metastable state. Of special interest is whether these states are associated with antiferroelectricity.

\section{Thermal Depolarization and Dielectric Relaxation}

We examined changes in structure and molecular motion during heating by thermal depolarization. Di-



Figure 10. Temperature dependence of remanent polarization $P_{\mathrm{r}}$ and real permittivity $\varepsilon^{\prime}$ of $\operatorname{VDF}(37) / \operatorname{TrFE}(63)$ and $\operatorname{VDF}(47) / \operatorname{TrFE}(53)$ copolymers.

electric relaxation was measured to study molecular motion. Figure 10 shows plots of remanent polarization $P_{\mathrm{r}}$ imparted at $-90^{\circ} \mathrm{C}$ and the real component of complex permittivity $\varepsilon^{\prime}$ at $1 \mathrm{kHz}$ as functions of temperature. For the $47 / 53 \mathrm{~mol} \%$ copolymer, $P_{\mathrm{r}}$ decreases first gradually, then rapidly above $20^{\circ} \mathrm{C}$ and finally disappears at $55^{\circ} \mathrm{C}$. The rather rapid decrease indicates a transition from the ferroelectric polar phase to non-polar state.

$\varepsilon^{\prime}$ shows a peak at $70^{\circ} \mathrm{C}$. Measurement of dielectric frequency spectra shows dipolar motion at $1-100 \mathrm{MHz}$ frequency. VDF-rich copolymers exhibit much sharper peak due to a dielectric anomaly associated with the ferroelectric-to-paraelectric transition and whose position defines the Curie point $T_{\mathrm{c}} \cdot{ }^{9}$ Although the peak is much broader, an analogous dipolar motion and transition into a paraelectric phase may take place in the $47 / 53 \mathrm{~mol} \%$ copolymer. Loss of remanent polarization occurs prior to dipolar motion in this copolymer, in contrast to VDF-rich copolymers where these events occur simultaneously.

The $37 / 63 \mathrm{~mol} \%$ copolymer exhibited essentially the same behavior except that transition took place over a broader temperature range than for the $47 / 53 \mathrm{~mol} \%$ copolymer. We determined transition temperatures as those where the rate of decrease in $P_{\mathrm{r}}$ and increase in $\varepsilon^{\prime}$ are maximum. These temperatures separate the ferroelectric, intermediate and paraelectric phases as shown by the dashed lines in Figure 10. The intermediate phase can be described as a non-polar and non-motional state (the antiferroelectric-like phase).

The ferroelectric phase of a VDF-rich copolymer consists of all-trans molecules packed in parallel. Molecular dipoles are thus aligned in one direction to form large spontaneous polarization $\left(>100 \mathrm{mC} \mathrm{m}^{-2}\right)$. As TrFE becomes a major component of the copolymer, the all-trans conformation is favored but ferroelectricity is obscured and lost in PTrFE. For the VDF/TrFE 37/63 copolymer, Tashiro et al. ${ }^{3}$ reported a transition between 
cooled and high temperature phases using X-ray diffraction and IR spectra. They found the transition over a very wide temperature range. This paper demonstrates that the broad transition can be separated into two transitions for the copolymers with composition $37 / 63$ and $47 / 53 \mathrm{~mol} \%$ that possess a non-polar intermediate phase.

Such a phase is possibly antiferroelectric. If all-trans molecules are packed in an anti-parallel fashion, an anti-polar phase consisting of two sublattices opposite in polarity results. If such an anti-polar phase is transformed by application of an electric field or cooling, we may call it antiferroelectric. However, such anti-polar arrangement is not yet confirmed experimentally. No difference in the extinction rule was observed between ferroelectric and intermediate phases because both phases give only a few $\mathrm{X}$-ray reflections.

Another possibility for such a non-polar state arises from a multi-domain structure in crystalline lamellae whose spontaneous polarization is randomly oriented. Uniaxially-drawn and well-annealed copolymer samples consist of large lamellae regularly stacked along the draw direction. ${ }^{10,11}$ Such a structure results in a very small fraction of inter-lamellar irregular regions which may cause a depolarization field in the lamella to diminish macroscopic polarization. Thus, we can impart fully saturated polarization that remains constant for years in VDF-rich copolymers. However, such a depolarization field does exist unless the sample has $100 \%$ crystallinity. Because TrFE-rich copolymers have a large lattice constants $a^{\prime}$ and $b^{3}$ and give diffuse X-ray diffraction patterns, their chains are packed loosely in crystals. Therefore, even a small depolarization field may cause a multi-domain structure to minimize the depolarization field. One possible structure for this state is the cooled phase proposed by Tashiro et al. ${ }^{3}$ In this phase, crystals are composed of domains separated by twin boundaries and the direction of polarization differs among domains.

\section{CONCLUSION}

Switching measurements were made for $37 / 63$ and $47 / 53 \mathrm{~mol} \% \mathrm{VDF} / \mathrm{TrFE}$ copolymers and polarization reversal was noted to occur in two steps through a non-polar state in parallel with double hysteresis loops: fast depolarization and slow re-polarization. The time constants were determined over a broad range of temperature and applied electric fields. Thermal depolarization and dielectric relaxation measurements characterized the non-polar state as antiferroelectric-like between low temperature ferroelectric and high temperature paraelectric phases. The structure of such a phase is discussed in terms of anti-parallel packing of all-trans molecules or micro-domains that divide a crystalline lamella into regions with distributed spontaneous polarization.

Acknowledgments. The authors are grateful to Daikin Industries, Ltd. for providing the copolymer samples.

\section{REFERENCES}

1. T. Furukawa, Phase Transitions, 18, 143 (1989).

2. K. Tashiro and M. Kobayashi, Phase Transitions, 18, 213 (1989)

3. K. Tashiro, K. Takano, M. Kobayashi, Y. Chatani, and H. Tadokoro, Ferroelectrics, 57, 297 (1984).

4. A. J. Lovinger, G. T. Davis, M. G. Broadhurst, and T. Furukawa, Macromolecules, 15, 323 (1982).

5. T. Horiuchi, K. Matsushige, and T. Takemura, Jpn. J. Appl. Phys., 25, L465 (1986).

6. Koizumi, Y. Murata, and H. Tsunashima, IEEE Trans. Elect. Insul., EI-21, 543 (1986).

7. Y. Oka, N. Koizumi, and Y. Murata, J. Polym. Sci., B: Polym. Phys., 24, 2059 (1986).

8. T. Furukawa, M. Date, M. Ohuchi, and A. Chiba, J. Appl. Phys., 56, 1481 (1984).

9. T. Furukawa, M. Ohuchi, A. Chiba, and M. Date, Macromolecules, 17, 1384 (1984).

10. T. Sasaki, A. Funato, K. Kubo, and A. Chiba, Jpn. J. Appl. Phys., 34, 3117 (1995).

11. H. Kodama, Y. Takahashi, and T. Furukawa, Ferroelectrics, 203 435 (1997). 